\title{
Cooling and Amplifying Micro-Mechanical Motion Using Light
}

\author{
Kerry Vahala \\ Mail Stop 128-95 Caltech, Pasadena, California 91125; vahala@caltech.edu
}

\begin{abstract}
Progress in the subject of cavity optomechanics will be described. Micromechanical oscillators to microwave rates, as well as progress directed towards cooling to the quantum ground state will be reviewed.

Recent years have witnessed a series of developments at the intersection of two, previously distinct subjects. Optical (micro-) cavities [1] and micro (nano-) mechanical resonators [2], each a subject in their own right with a rich scientific and technological history, have, in a sense, become entangled experimentally by the underlying mechanism of optical forces. These forces and their related physics have been of major interest in the field of atomic physics for over 5 decades [3-5], and the emerging opto-mechanical context for these forces has many parallels with this field. There is also a rich theoretical history that considers the implications of optical forces in this new context [6-9]. Despite this theoretical promise, the manifestations of these forces on micro-mechanical objects have only recently become an experimental reality [10]. We will review recent demonstrations of both mechanical amplification and cooling by radiation pressure forces in micron-scale toroidal resonators and also in silicon cantilever-based resonators. These devices contain high-Q optical modes in coexistence with high- $Q$ mechanical modes. Resonantly enhanced optical forces couple these mechanical and optical degrees of freedom, creating two distinct dynamical regimes. In the first, mechanical amplification can overcome intrinsic loss to induce regenerative mechanical oscillation up to microwave rates [12]. In the second, mechanical cooling to low temperatures is possible (sub Kelvin cooling from room temperature has been demonstrated). After discussing progress directed to ground-state cooling of a macroscale mechanical oscillator $[13,14]$, the possible future directions of this emerging field of cavity opto-mechanics will be considered.
\end{abstract}

[1] K. Vahala, "Optical Microcavities," Nature, vol. 424, No. 6950, August 2003.

[2] K. Schwab and M L Roukes, "Putting Mechanics into Quantum Mechanics," Physics Today, July 2005.

[3] A. Ashkin, Physical Review Letters 24, 156 (1970).

[4] T. W. Hänsch, A. L. Schawlow, Optics Communications 13, 68 (1975).

[5] D. Wineland, H. Dehmelt, Bulletin of the American Physical Society 20, 637 (1975).

[6] V. B. Braginsky, Measurement of Weak Forces in Physics Experiments (University of Chicago Press, Chicago, (1977), pp.

[7] C. M. Caves, Physical Review D 23, 1693 (1981).

[8] V. B. Braginsky, S. P. Vyatchanin, Phys. Lett. A, 293, 228 (2002).

[9] S. Mancini et. al., Phys. Rev Lett., 88, no. 12, 120401-1 (2002); Marshall, W. et. al. Phys. Rev. Lett., 91, 130401 (2003).

[10] T. Kippenberg and K. Vahala, "Cavity Optomechanics," Optics Express Review, Dec 10, 2007.

[11] D. K. Armani, et. al. Nature, 421, pp. 925-929, 27 February (2003).

[12] T. J. Kippenberg, et. al. Phys. Rev. Lett. 95, 033901, 2005. ; T. Carmon, et. al., Phys. Rev. Lett., 94, 223902, June 2005.; H. Rokhsari, et. al. Optics Express, 13, No. 14, July 2005.

[13] S. Gigan, H.R. et. al., Nature (London) 444, 67 (2006); O. Arcizet, et. al., Nature (London) 444, 71 (2006), A. Schliesser, et. al. Phys. Rev. Lett., 97, 243905, Dec 15, (2006).

[14] Kleckner, D.\& Bouwmeester, D., Nature 432, 75-78 (2006).

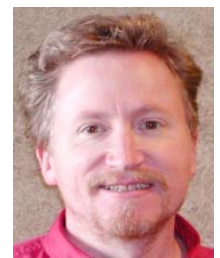

Kerry Vahala is Ted and Ginger Jenkins Professor of Information Science and Technology and Professor of Applied Physics at Caltech. He also received his Ph. D. (85) in Applied Physics at Caltech. His research on micro-resonators has led to wafer-based devices operating in the $Q$ regime above 100 million and has also provided low-loss methods for coupling directly to optical fiber. These devices have enabled micro-scale Raman and Parametric sources as well as cavity QED ona-chip systems. His current research is focused on a range of opto-mechanical phenomena associated with radiation pressure in microresonators. Vahala is a Fellow of the Optical Society of America, was the first recipient of the Richard P. Feynman Hughes Fellowship, and is also a recipient of an Alexander Von Humboldt Research Award. He received both the Presidential Young Investigator and Office of Naval Research Young Investigator Awards; and has been a topical editor for the Journal of the Optical Society of America and Photonics Technology Letters. He was also program co-chair for CLEO 99 and General Chair for CLEO 2001. 